Research Paper

\title{
Pre-treatment Serum Lactate Dehydrogenase is Predictive of Survival in Patients with Nasopharyngeal Carcinoma Undergoing Intensity-Modulated Radiotherapy
}

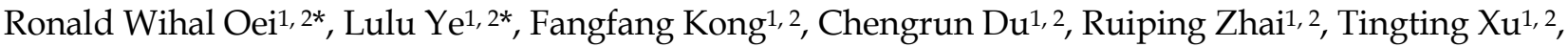 \\ Chunying Shen ${ }^{1,2}$, Xiaoshen Wang1, 2, Xiayun He1, 2, Lin Kong1, 2, Chaosu Hu1,2, Hongmei Ying1,2匹 \\ 1. Department of Radiation Oncology, Fudan University Shanghai Cancer Center, Shanghai, 200032, P.R China; \\ 2. Department of Oncology, Shanghai Medical College, Fudan University, Shanghai, 200032, P.R China. \\ *Ronald Wihal Oei and Lu-Lu Ye made equal contribution to this work. \\ $\triangle$ Corresponding author: Professor Hongmei Ying; Department of Radiation Oncology, Fudan University Shanghai Cancer Center, 270 Dongan Road, \\ Shanghai, 200032, P.R China; Telephone: +86-21-64175590; Fax: +86-21-6417477; E-mail: yinghongmei2013@163.com \\ (c) Ivyspring International Publisher. This is an open access article distributed under the terms of the Creative Commons Attribution (CC BY-NC) license \\ (https://creativecommons.org/licenses/by-nc/4.0/). See http://ivyspring.com/terms for full terms and conditions.
}

Received: 2017.08.01; Accepted: 2017.10.24; Published: 2018.01.01

\begin{abstract}
Objective: To analyze the prognostic value of pre-treatment serum lactate dehydrogenase (SLDH) level in patients with nasopharyngeal carcinoma (NPC) receiving intensity-modulated radiotherapy (IMRT) with or without chemotherapy.

Methods: From January 2010 to March 2013, 427 eligible patients were reviewed. Pre-treatment SLDH level was measured within 2 weeks prior to treatment. Receiver operating characteristic (ROC) curve analysis was performed to select the optimal cutoff point. The impact of pre-treatment SLDH on overall survival (OS), progression-free survival (PFS) and distant metastasis-free survival (DMFS) were analyzed using Kaplan-Meier method and Cox proportional hazards model. Further propensity score matching was carried out to adjust bias.

Results: The optimal cutoff point of $168.5 \mathrm{IU} / \mathrm{L}$ was selected based on ROC curve analysis. Multivariate analysis showed that high pre-treatment SLDH level was an independent prognostic factor for OS $(P=0.001)$, PFS $(P=0.004)$ and $D M F S(P=0.001)$. After propensity score matching was performed, it remained to be significantly associated with poor OS $(P=0.009)$, $P F S(P=0.015)$ and DMFS $(P=0.008)$ in the adjusted model.

Conclusion: High pre-treatment SLDH level predicts poor survival in patients with NPC treated with IMRT-based therapy. As a routinely performed biomarker, pre-treatment SLDH can be utilized in combination with current Tumor-Node-Metastasis staging to predict survival and to plan a personalized treatment in these patients.
\end{abstract}

Key words: Lactate dehydrogenase, Nasopharyngeal carcinoma, Intensity-modulated radiotherapy, Survival.

\section{Introduction}

Nasopharyngeal carcinoma (NPC) is a malignancy with extremely unbalanced racial and geographic distribution. Although being rare globally, the incidence rates are notably high in South-Eastern Asia and South-Eastern China ${ }^{1}$. In Mainland China, the incidences of NPC are remarkably high in five provinces, including: Guangdong, Hainan, Guangxi, Hunan and Fujian ${ }^{2}$. Compared to other head and neck cancers (HNC), radiotherapy with or without chemotherapy is the primary treatment modality of NPC. In the era of conventional external beam radiotherapy, locoregional recurrence and distant 
metastasis contributed equally to the treatment failures of $\mathrm{NPC}^{3,4}$. However, the widespread application of intensity-modulated radiotherapy (IMRT) over the past decades has improved the locoregional control of NPC significantly, but no similar effect found for distant metastasis 5,6 . Consequently, distant metastasis is the main cause of treatment failures among NPC patients nowadays ${ }^{7,8}$. Therefore, it is crucial to have an ability to identify NPC patients with high risk of distant metastasis and subsequent poor prognosis before treatment in order to provide early intervention and better treatment decision.

Tumor-Node-Metastasis (TNM) staging system are currently the most broadly used parameters in treatment decision and prediction of treatment outcomes for NPC patients. However, current TNM staging system does not take biological diversity of the tumor into consideration ${ }^{9}$. Recently, more and more biological markers were identified as prognostic factors for NPC, including: plasma Epstein-Barr virus deoxyribonucleic acid (EBV-DNA) concentration ${ }^{10,11}$, Beclin $1^{12}$ and serum lactate dehydrogenase (SLDH) level13-15. Among these, SLDH level has some advantages in accessibility and cost-efficacy. Although being frequently investigated, most of the studies on SLDH level and its prognostic value in NPC patients derived from high-incidence areas, including Guangdong14,16, Guangxi ${ }^{13}$ and Fujian ${ }^{15}$ provinces. Therefore, new research from middle- and low-incidence areas are urgently needed to reduce selection and reporting bias and subsequently increase the reliability of SLDH level as a prognostic indicator in NPC.

Therefore, a total of 427 non-metastatic NPC patients treated with IMRT-based therapy were included in this retrospective study. The objective of our study was to evaluate the prognostic significance of pre-treatment SLDH in NPC patients receiving IMRT in Eastern China. Furthermore, propensity score matching was carried out in order to minimize the effect of confounders.

\section{Materials and Methods}

\section{Study population}

This retrospective study was approved by the Institutional Review Board of Fudan University Shanghai Cancer Center. The study was run in accordance with institutional policy and the principles of Declaration of Helsinki and its amendments.

A total of 427 newly diagnosed NPC patients treated with IMRT-based therapy at Fudan University Shanghai Cancer Center between January 2010 and
March 2013 were included in this retrospective analysis. The inclusion criteria were: (1) 16 years old and above; (2) Histologically confirmed non-keratinizing and/or undifferentiated nasopharyngeal carcinoma (World Health Organization type II/III); (3) Karnofsky performance status scale $\geq 80$; (4) No evidence of distant metastasis and concomitant malignancies at diagnosis; (5) Adequate cardiac (New York Heart Association Functional Capacity $\leq \mathrm{II}$ ), liver (total bilirubin and alanine aminotransferase level $\leq 1.5 \mathrm{x}$ the upper reference limit) and renal (creatinine level $\leq 1.5 x$ the upper reference limit) function; (6) Completion of prescribed treatment; (7) Complete baseline data of biochemistry blood test, including serum lactate dehydrogenase level. TNM stage of the patients were classified according to the seventh edition of American Joint Committee on Cancer (AJCC) staging system.

\section{Pre-treatment workup}

All patients underwent full pre-treatment workup, including clinical history, physical examination, contrast-enhanced magnetic resonance imaging (MRI) or computed tomography (CT) scan of nasopharynx and neck, plain chest $\mathrm{CT}$, abdominal sonography, a whole body bone scan by single-photon emission computed tomography (SPECT), complete blood count and serum biochemistry test.

Peripheral fasting blood sample was taken through venipuncture from each patient to assess the pre-treatment serum lactate dehydrogenase level. It was obtained within 2 weeks before any kind of treatment started. Serum lactate dehydrogenase level was measured using chemistry analyzer cobas 8000 (Roche, Rotkreuz, Switzerland).

\section{Treatment protocol}

All patients were treated with IMRT. The prescribed dose was 66-70.4 Gy to the planning target volume (PTV) of the gross tumor volume of nasopharynx (GTVnx), 66-70 Gy to the PTV of the gross tumor volume of metastatic neck lymph nodes (GTVnd), 60 Gy to the PTV of the high-risk region defined as clinical target volume 1 (CTV1) and 54 Gy to the PTV of the low-risk region defined as clinical target volume 2 (CTV2). The planning target volumes were contoured by adding 5 millimeters and 3 millimeters to GTV and CTV respectively. The prescribed radiation dose was delivered in 30-35 fractions.

Based on the standardized treatment of our institution, radiotherapy alone was only recommended for patients with stage I, while 
combined chemoradiotherapy was suggested for patients with stage II and above. To be more specific, concurrent chemoradiotherapy was prescribed for patients with stage II and either concurrent chemoradiotherapy or neoadjuvant+adjuvant chemotherapy for patients with stage III and above.

The regimens prescribed for neoadjuvant and adjuvant chemotherapy were mostly: (1) TPF regimen: docetaxel $60 \mathrm{mg} / \mathrm{m}^{2} /$ day on day $1+$ cisplatin $25 \mathrm{mg} / \mathrm{m}^{2} /$ day on day $1-3+5$-fluorouracil $0.5 \mathrm{~g} / \mathrm{m}^{2} /$ day on day $1-3,(2)$ TP regimen: docetaxel 60 $\mathrm{mg} / \mathrm{m}^{2} /$ day on day $1+$ cisplatin $25 \mathrm{mg} / \mathrm{m}^{2} /$ day on day 1-3, (3) GP regimen: gemcitabine $1 \mathrm{~g} / \mathrm{m}^{2} /$ day on day $1,8+$ cisplatin $25 \mathrm{mg} / \mathrm{m}^{2} /$ day on day $1-3$. The first-line chemotherapy regimens were TPF and TP regimens. However, GP regimen was administered if patients had following clinical history: peptic ulcer, upper gastrointestinal tract bleeding, cardiac disease, diabetes and food/drug allergy. Moreover, chemotherapy was not prescribed to following patients: (1) 70 years old and above; (2) The existence of one or more certain comorbidities including, but not limited to, active tuberculosis and low blood counts. Concurrent chemotherapy was consisted of cisplatin $80 \mathrm{mg} / \mathrm{m}^{2}$. Chemotherapy was administered every 3 weeks for 2 to 3 cycles.

\section{Follow-up and clinical endpoints}

Following completion of treatment, all patients were followed-up at 3-month intervals during the first 2 years and every 6 to 12 months thereafter. Physical examination, including indirect nasopharyngoscopy and palpation of the neck lump examination, was performed at every clinic visit. Apart from that, contrast-enhanced MRI of nasopharnyx, plain chest CT scan and abdominal sonography were done at 6-month interval. Further test would be taken if any sign of locoregional or distant failures were detected. Patients with confirmation of recurrence or metastasis were undergoing further treatment.

The primary endpoint of this study was overall survival (OS), while the secondary endpoints were progression-free survival (PFS) and distant metastasis-free survival (DMFS). OS was defined as the time interval between the initial treatment time and death from any cause. PFS was defined as the time from initial treatment to the date of disease progression or death from any cause. DMFS was defined as the elapsed time between the date of initial treatment and the first appearance of distant metastasis.

\section{Statistical analysis}

The Statistical Packages for Social Sciences version 22.0 (IBM, Armonk, NY) were used in data analysis. In current study, receiver operating characteristics (ROC) curve was performed to calculate the most appropriate cutoff point for SLDH level. $X^{2}$ test (or Fischer's exact test, if indicated) was used to test the baseline balance between low SLDH and high SLDH groups. Actuarial survival rates for OS, PFS and DMFS were obtained using Kaplan-Meier method. In addition, differences in survival between groups were calculated using log-rank test. Univariate and multivariate analysis were performed using Cox proportional hazards model (multivariate analysis consisted of variables with $\mathrm{P}$ value $<0.05$ in univariate analysis). Proportional hazard assumption was assessed using log-minus-log plots. Any result with two-sided P value $<0.05$ was considered statistically significant.

In addition, propensity score matching was performed using $\mathrm{R}$ version 3.4.0 (The R Foundation of Statistical Computing, Vienna, Austria) to adjust for bias and confounding. Variables used to calculate the propensity score index were: age, sex, tumor classification, nodal classification, TNM staging and radiation dose. This was performed using nearest neighbor 1:1 matching in MatchIt package.

\section{Results}

\section{Patient characteristics}

Baseline characteristics of 427 NPC patients reviewed are provided in table 1 . The median age was 48 years which ranged from 17 to 82 years old. There were 307 males $(71.9 \%)$ and 120 females (28.1\%) with a sex ratio of 2.6:1. According to the seventh edition of AJCC staging manual, there were $9(2.1 \%), 80(18.7 \%)$, $208(48.7 \%)$ and $130(30.4 \%)$ patients with stage I, II, III and IV respectively. Among these patients, 368 patients $(86.2 \%)$ received combined chemoradiotherapy. Furthermore, the demographic profiles of these patients are presented in table 2. As can be seen in the table, around three-quarters of the patients came from Zhejiang province, Jiangsu province and Shanghai municipality. Moreover, if Anhui province and Jiangxi province are added, these five provinces made up more than $90 \%$ of the entire population, which means most of the patients originated in Shanghai and surrounding areas.

On the whole, the median follow-up time was 67.5 months which ranged from 4.8 to 85.5 months. During this time period, 57 patients (13.3\%) experienced locoregional recurrence, 64 patients $(15.0 \%)$ developed distant metastasis and 64 patients $(15.0 \%)$ were dead. The 5-year OS, PFS and DMFS were $85.8 \%, 76.0 \%$ and $84.8 \%$, respectively. 


\section{Receiver operating characteristic curve analysis and the distribution of baseline characteristics in the study population}

With OS as an endpoint, a cutoff value of 168.5 IU/L for pre-treatment SLDH was obtained through ROC curve analysis. There were 249 (58.3\%) patients with pre-treatment SLDH below 168.5 IU/L and 178 $(41.7 \%)$ patients above this point. The sensitivity and specificity of this cutoff value were $64.1 \%$ and $62.3 \%$ respectively. As shown in figure 1, the area under the curve was 0.643 with $95 \%$ confidence interval (CI) ranged from 0.568 to 0.717 and $P$ value $<0.001$.

Table 1. Baseline characteristics of 427 patients with nasopharyngeal carcinoma

\begin{tabular}{|c|c|c|c|c|c|}
\hline \multirow[t]{2}{*}{ Characteristics } & \multirow[t]{2}{*}{$\mathrm{N}$} & \multirow[t]{2}{*}{$\%$} & \multicolumn{2}{|c|}{ SLDH Level (IU/L) } & \multirow[t]{2}{*}{ P value } \\
\hline & & & $<168.5$ & $\geq 168.5$ & \\
\hline Age (years) & & & & & 0.162 \\
\hline$<60$ & 340 & 79.6 & 204 & 136 & \\
\hline$\geq 60$ & 87 & 20.4 & 45 & 42 & \\
\hline Sex & & & & & 0.029 \\
\hline Male & 307 & 71.9 & 189 & 118 & \\
\hline Female & 120 & 28.1 & 60 & 60 & \\
\hline Tumor classification ${ }^{b}$ & & & & & 0.529 \\
\hline $\mathrm{T} 1-\mathrm{T} 2$ & 214 & 50.1 & 128 & 86 & \\
\hline T3-T4 & 213 & 49.9 & 121 & 92 & \\
\hline Nodal classification ${ }^{b}$ & & & & & 0.004 \\
\hline N0-N1 & 196 & 45.9 & 129 & 67 & \\
\hline N2-N3 & 231 & 54.1 & 120 & 111 & \\
\hline TNM stageb & & & & & 0.007 \\
\hline I-II & 89 & 20.8 & 63 & 26 & \\
\hline III-IV & 338 & 79.2 & 186 & 152 & \\
\hline Treatment modality & & & & & 0.333 \\
\hline RT alone & 59 & 13.8 & 31 & 28 & \\
\hline CRT & 368 & 86.2 & 218 & 150 & \\
\hline
\end{tabular}

Abbreviations: CRT, combined chemoradiotherapy; IU, international unit; L, liter; $\mathrm{RT}$, radiotherapy; SLDH, serum lactate dehydrogenase; TNM, tumor-node-metastasis.

aChi-square $\left(\chi^{2}\right)$ test, $P<0.05$

bTumor-node-metastasis staging system proposed by the American Joint

Committee on Cancer (7th edition).
As depicted in table 1 , there were significant differences in the distribution of sex, nodal classification and TNM stage observed in the study cohort. There was a higher proportion of female patients with high pre-treatment SLDH $(\geq 168.5 \mathrm{IU} / \mathrm{L})$. Besides, advanced nodal classification and TNM stage were more frequent in patients with high pre-treatment SLDH.

\section{Univariate and multivariate analysis}

In comparison with low pre-treatment SLDH group, patients with high pre-treatment SLDH had significantly lower OS (5-year OS: 78.5\% versus $91.0 \%$, P<0.001), PFS (5-year PFS: 68.4\% versus 81.4\%, $\mathrm{P}=0.001$ ) and DMFS (5-year DMFS: $77.1 \%$ versus $90.3 \%, \mathrm{P}<0.001$ ) (Figure 2).

Table 2. Demographic characteristics of 427 patients with nasopharyngeal carcinoma

\begin{tabular}{lll}
\hline Region & $\mathrm{N}$ & $\%$ \\
\hline Anhui province & 39 & 9.1 \\
Fujian province & 13 & 3.0 \\
Gansu province & 3 & 0.7 \\
Guangxi zhuang autonomous region & 2 & 0.5 \\
Hainan province & 2 & 0.5 \\
Heilongjiang province & 2 & 0.5 \\
Henan province & 2 & 0.5 \\
Hubei province & 2 & 0.5 \\
Hunan province & 2 & 0.5 \\
Jiangsu province & 75 & 17.5 \\
Jiangxi province & 42 & 9.8 \\
Shanghai municipality & 97 & 22.7 \\
Sichuan province & 3 & 0.7 \\
Yunnan province & 1 & 0.2 \\
Zhejiang province & 142 & 33.3 \\
\hline
\end{tabular}

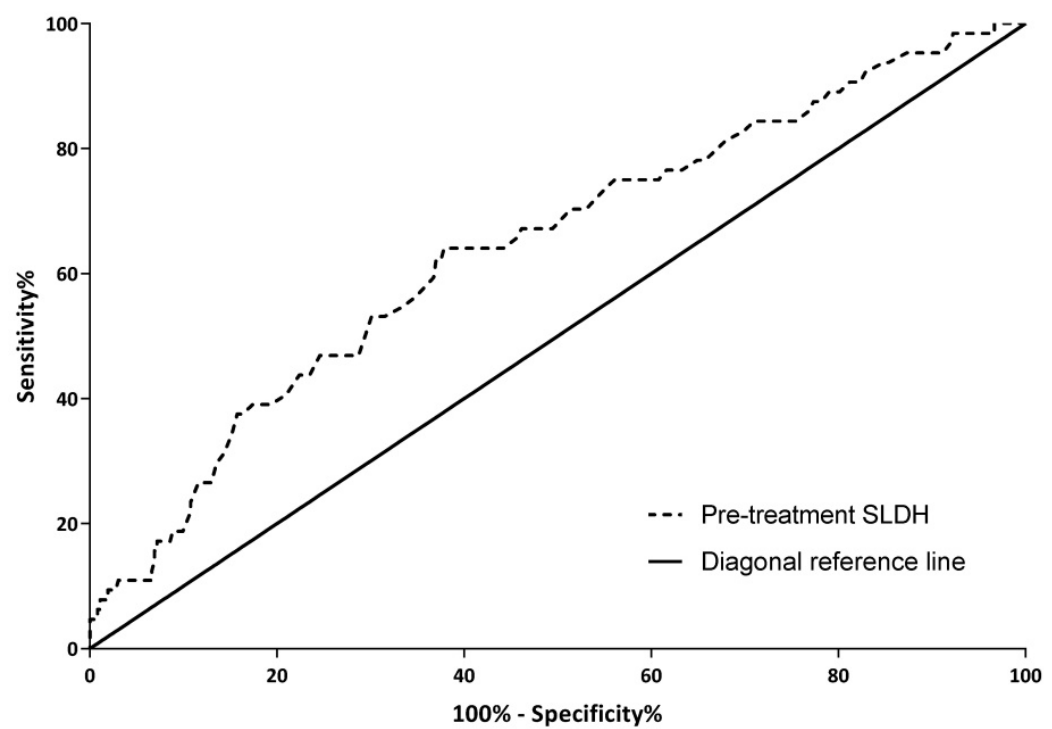

Figure 1 Receiver operating characteristic (ROC) curve for pre-treatment serum lactate dehydrogenase level based on overall survival 

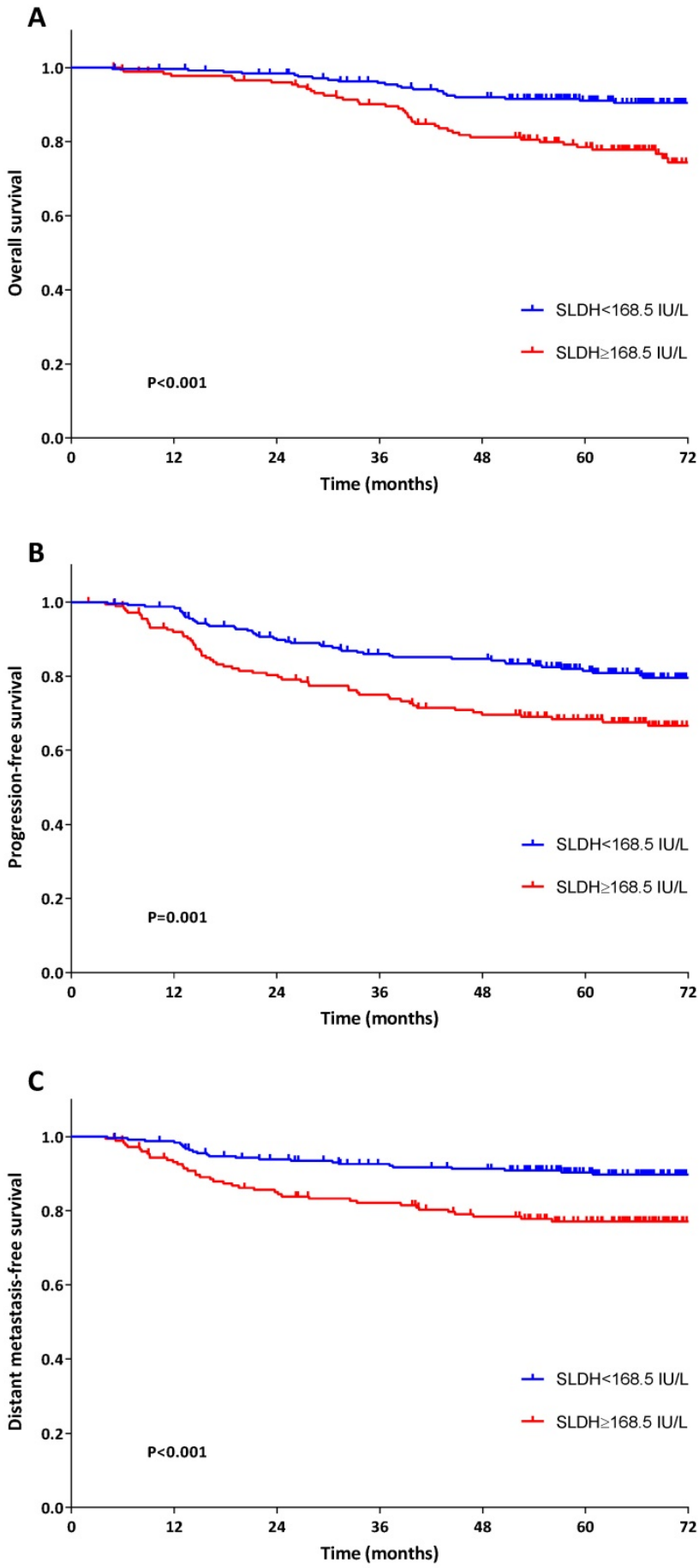

Figure 2 Kaplan-Meier survival plots for OS (A), PFS (B) and DMFS (C) stratified by pre-treatment serum lactate dehydrogenase

In Cox univariate analysis, greater age $(\geq 60$ versus $<60$ years), advanced nodal classification (N2-N3 versus N0-N1) and high pre-treatment SLDH ( $\geq 168.5 \mathrm{IU} / \mathrm{L}$ versus $<168.5 \mathrm{IU} / \mathrm{L})$ had significant association with poor OS. The hazard ratio (HR) of these variables on decreased OS was 2.469 (95\% CI: 1.481-4.116, $\mathrm{P}=0.001), 1.908$ (95\% CI: 1.130-3.220, $\mathrm{P}=0.016)$ and 2.704 (95\% CI: 1.622-4.507, $\mathrm{P}<0.001$ ), respectively (Table 3 ). In terms of PFS, the following variables were found to be significantly correlated with worse PFS: advanced tumor classification (HR: 1.675, 95\% CI: 1.137-2.467, $\mathrm{P}=0.009)$, advanced nodal classification (HR: 1.718, 95\% CI: 1.159-2.547, $\mathrm{P}=0.007)$, late TNM stage (HR: 1.940, 95\% CI: 1.106-3.405, $\mathrm{P}=0.021)$ and high pre-treatment SLDH (HR: 1.867, 95\% CI: 1.276-2.731, $\mathrm{P}=0.001$ ) (Table 4). With regard to DMFS, advanced tumor classification (HR: 1.747, 95\% CI: 1.053-2.898, $\mathrm{P}=0.031)$, advanced nodal classification (HR: 2.747, 95\% CI: 1.559-4.840, P<0.001), late TNM stage (HR: 2.663, 95\% CI: 1.149-6.174, $\mathrm{P}=0.022$ ) and high pre-treatment SLDH (HR: 2.542, 95\% CI: 1.532-4.218, $\mathrm{P}<0.001)$ were identified to be predictive of distant metastasis (Table 5).

Table 3. Univariate and multivariate analysis for overall survival of 427 patients with nasopharyngeal carcinoma

\begin{tabular}{|c|c|c|c|c|}
\hline \multirow[t]{2}{*}{ Variables } & \multicolumn{2}{|l|}{ Univariate analysis } & \multicolumn{2}{|c|}{ Multivariate analysis } \\
\hline & HR $(95 \%$ CI) & $P$ value ${ }^{a}$ & HR $(95 \% \mathrm{CI})$ & P value \\
\hline Age (years) & & 0.001 & & 0.001 \\
\hline$<60$ & Ref & & Ref & \\
\hline$\geq 60$ & $2.469(1.481-4.116)$ & & $\begin{array}{l}2.320 \\
(1.390-3.874)\end{array}$ & \\
\hline Sex & & 0.596 & & \\
\hline Male & Ref & & & \\
\hline Female & $0.858(0.487-1.512)$ & & & \\
\hline Tumor classification ${ }^{b}$ & & 0.239 & & \\
\hline $\mathrm{T} 1-\mathrm{T} 2$ & Ref & & & \\
\hline T3-T4 & $1.345(0.821-2.205)$ & & & \\
\hline Nodal classification ${ }^{b}$ & & 0.016 & & 0.037 \\
\hline N0-N1 & Ref & & Ref & \\
\hline N2-N3 & $1.908(1.130-3.220)$ & & $\begin{array}{l}1.751 \\
(1.034-2.964)\end{array}$ & \\
\hline TNM stage ${ }^{b}$ & & 0.134 & & \\
\hline I-II & Ref & & & \\
\hline III-IV & $1.716(0.848-3.472)$ & & & \\
\hline Serum LDH (IU/L) & & $<0.001$ & & 0.001 \\
\hline$<168.5$ & Ref & & Ref & \\
\hline$\geq 168.5$ & $2.704(1.622-4.507)$ & & $\begin{array}{l}2.425 \\
(1.449-4.060)\end{array}$ & \\
\hline
\end{tabular}

Abbreviations: CI, confidence interval; HR, hazard ratio; IU, international unit; L, liter; Ref, reference.

aCox proportional hazards model, $\mathrm{P}<0.05$ were in bold.

bTumor-node-metastasis staging system according to the American Joint

Committee on Cancer ( $7^{\text {th }}$ edition).

All variables with $P$ value less than 0.05 in univariate analysis were included in multivariate Cox proportional hazards regression analysis. Age (HR: 2.320, 95\% CI: 1.390-3.874, $\quad \mathrm{P}=0.001)$, nodal classification (HR: 1.751, 95\% CI: 1.034-2.964, $\mathrm{P}=0.037$ ) and pre-treatment SLDH (HR: 2.425, 95\% CI: 1.449-4.060, $\mathrm{P}=0.001$ ) were an independent prognostic factor for OS (Table 3). As regards PFS, advanced tumor classification (HR: 2.018, 95\% CI: 1.246-3.269, $\mathrm{P}=0.004$ ), advanced nodal classification (HR: 2.001, 95\% CI: 1.213-3.300, $\mathrm{P}=0.007)$ and high pre-treatment SLDH (HR: 1.768, 95\% CI: 1.205-2.594, $\mathrm{P}=0.004$ ) remained to be independently associated with poor PFS (Table 4). Regarding DMFS, advanced tumor classification (HR: 2.216, 95\% CI: 1.235-3.977, $\mathrm{P}=0.008$ ), advanced nodal classification (HR: 3.309, 95\% CI: 
1.614-6.781, $\mathrm{P}=0.001$ ) and high pre-treatment SLDH (HR: 2.315, 95\% CI: 1.391-3.853, $\mathrm{P}=0.001$ ) were independently correlated with worse DMFS (Table 5).

\section{Propensity-matched analysis}

Propensity score matching (PSM) was performed in order to adjust bias to a greater degree. PSM was carried out by matching several variables, including: age, sex, tumor classification, nodal classification, TNM stage and radiation dose between high and low pre-treatment SLDH level. The clinical characteristics of new patient cohort $(n=356)$ is summarized in table 6 . In addition, the distribution of propensity scores of both groups before and after PSM can be seen in figure 3. Clearly, there were considerable discrepancy in the distribution of propensity scores between both groups before PSM, but the distributions then became very closely aligned after PSM performed.

Table 4. Univariate and multivariate analysis for progression-free survival of 427 patients with nasopharyngeal carcinoma

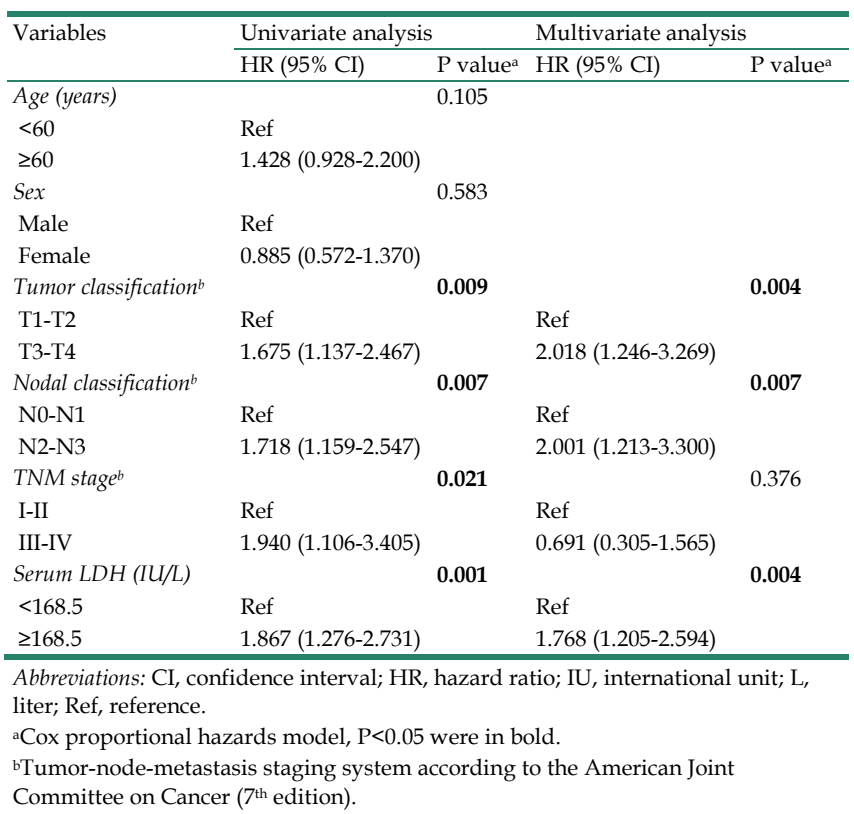

In subsequent unadjusted Cox regression analysis, pre-treatment SLDH was still significantly correlated with OS (HR: 2.065, 95\% CI: 1.220-3.495, $\mathrm{P}=0.007)$, PFS (HR: 1.684, 95\% CI: 1.118-2.535, $\mathrm{P}=0.013$ ) and DMFS (HR: 2.148, 95\% CI: 1.256-3.675, $\mathrm{P}=0.005$ ). After adjustment for sex (male versus female), age ( $<60$ years versus $\geq 60$ years), tumor classification (T1-T2 versus T3-T4), nodal classification (N0-N1 versus N2-N3) and radiotherapy dose (continuous variable), high pre-treatment SLDH remained as independent prognosticator of poor OS (HR: 2.042, 95\% CI: 1.203-3.464, P=0.008), PFS (HR: 1.663, 95\% CI: 1.103-2.507, $\mathrm{P}=0.015)$ and DMFS (HR: 2.058, 95\% CI: 1.200-3.528, $\mathrm{P}=0.009$ ) (Table 7).

\section{Discussion}

In the era of IMRT, NPC has gained significantly better treatment outcomes in terms of local and regional control, but unfortunately no such benefit found in distant metastasis control ${ }^{5,17}$. Moreover, some crucial problems still exist in current TNM staging system as the primary parameter used in treatment planning and survival prediction for NPC patients ${ }^{9}$. Therefore, identification of some biomarkers capable for prognosticating survival in these patients are urgently needed to optimize the current clinical management.

Table 5. Univariate and multivariate analysis for distant metastasis-free survival of 427 patients with nasopharyngeal carcinoma

\begin{tabular}{|c|c|c|c|c|}
\hline \multirow[t]{2}{*}{ Variables } & \multicolumn{2}{|l|}{ Univariate analysis } & \multicolumn{2}{|l|}{ Multivariate analysis } \\
\hline & HR $(95 \% \mathrm{CI})$ & Pvalue ${ }^{a}$ & $\mathrm{HR}(95 \% \mathrm{CI})$ & P value ${ }^{a}$ \\
\hline Age (years) & & 0.280 & & \\
\hline$<60$ & Ref & & & \\
\hline$\geq 60$ & $1.367(0.776-2.407)$ & & & \\
\hline $\operatorname{Sex}$ & & 0.936 & & \\
\hline Male & Ref & & & \\
\hline Female & $1.023(0.593-1.764)$ & & & \\
\hline Tumor classification ${ }^{b}$ & & 0.031 & & 0.008 \\
\hline T1-T2 & Ref & & Ref & \\
\hline T3-T4 & $1.747(1.053-2.898)$ & & $2.216(1.235-3.977)$ & \\
\hline Nodal classification ${ }^{b}$ & & $<0.001$ & & 0.001 \\
\hline N0-N1 & Ref & & Ref & \\
\hline N2-N3 & $2.747(1.559-4.840)$ & & $3.309(1.614-6.781)$ & \\
\hline TNM stage ${ }^{b}$ & & 0.022 & & 0.339 \\
\hline I-II & Ref & & Ref & \\
\hline III-IV & $2.663(1.149-6.174)$ & & $0.565(0.175-1.821)$ & \\
\hline Serum LDH (IU/L) & & $<0.001$ & & 0.001 \\
\hline$<168.5$ & Ref & & Ref & \\
\hline$\geq 168.5$ & $2.542(1.532-4.218)$ & & $2.315(1.391-3.853)$ & \\
\hline \multicolumn{5}{|c|}{$\begin{array}{l}\text { Abbreviations: CI, confidence interval; HR, hazard ratio; IU, international unit; L, } \\
\text { liter; Ref, reference. }\end{array}$} \\
\hline${ }^{\mathrm{a} C o x}$ proportional ha & zards model, $\mathrm{P}<0.05$ & were in $b$ & old. & \\
\hline $\begin{array}{l}\text { 'Tumor-node-metas } \\
\text { Committee on Cance }\end{array}$ & $\begin{array}{l}\text { is staging system ac } \\
\text { (7th edition). }\end{array}$ & ccording & to the American Jo & \\
\hline
\end{tabular}

Table 6. Baseline characteristics of 356 patients with nasopharyngeal carcinoma after propensity score matching

\begin{tabular}{|c|c|c|}
\hline Characteristics & $\mathrm{N}$ & $\%$ \\
\hline \multicolumn{3}{|l|}{ Age (years) } \\
\hline$<60$ & 276 & 77.5 \\
\hline$\geq 60$ & 80 & 22.5 \\
\hline \multicolumn{3}{|l|}{ Sex } \\
\hline Male & 241 & 67.7 \\
\hline Female & 115 & 32.3 \\
\hline \multicolumn{3}{|c|}{ Tumor classificationa } \\
\hline T1-T2 & 176 & 49.4 \\
\hline T3-T4 & 180 & 50.6 \\
\hline \multicolumn{3}{|c|}{ Nodal classification ${ }^{a}$} \\
\hline N0-N1 & 148 & 41.6 \\
\hline N2-N3 & 208 & 58.4 \\
\hline \multicolumn{3}{|l|}{ TNM stage ${ }^{a}$} \\
\hline I-II & 65 & 18.3 \\
\hline III-IV & 291 & 81.7 \\
\hline
\end{tabular}

Abbreviations: TNM, tumor-node-metastasis.

aTumor-node-metastasis staging system proposed by the American Joint

Committee on Cancer (7th edition). 
A

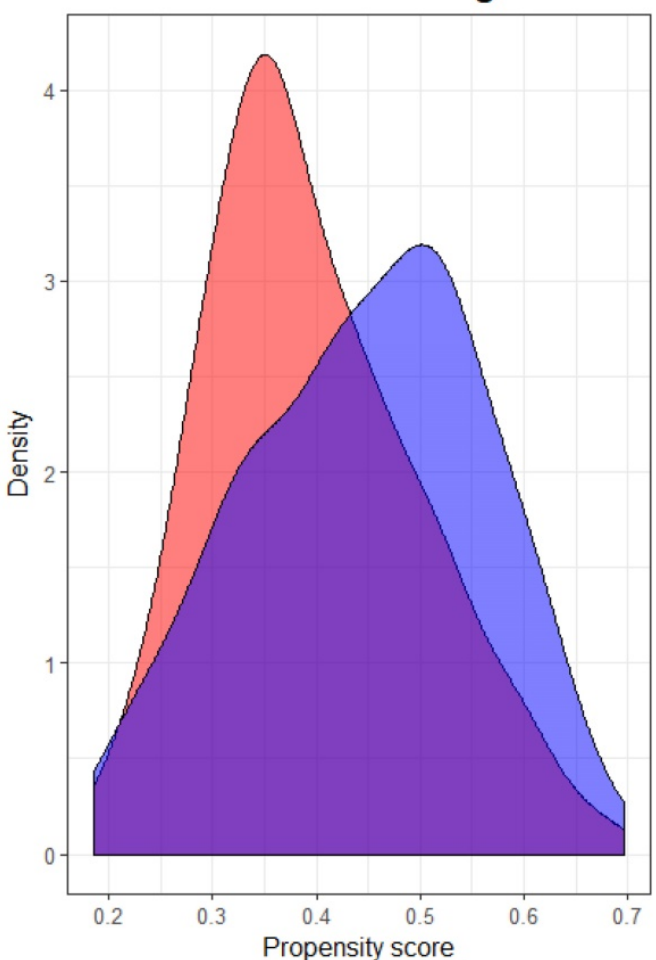

B

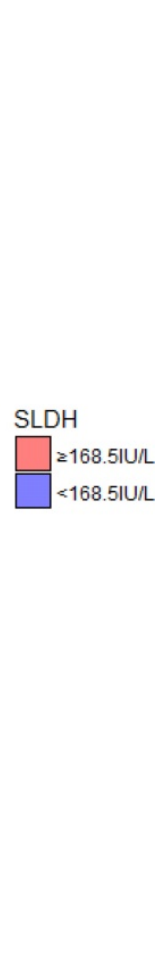

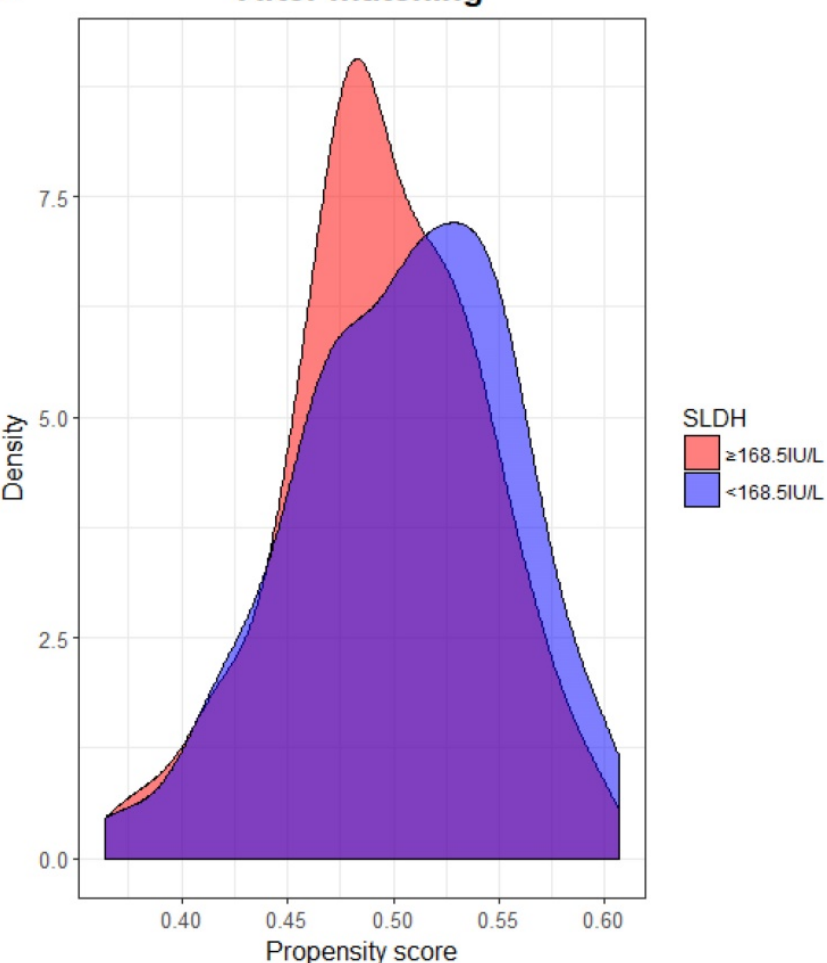

Figure 3 The distribution of propensity scores across serum lactate dehydrogenase (SLDH) level before (A) and after (B) propensity score matching.

Table 7. Cox regression analysis of the association between serum lactate dehydrogenase level and survival before and after propensity score matching

\begin{tabular}{|c|c|c|c|c|c|c|}
\hline & \multicolumn{2}{|l|}{ DMFS } & \multicolumn{2}{|l|}{ PFS } & \multicolumn{2}{|l|}{ OS } \\
\hline & HR $(95 \%$ CI) & $P$ value ${ }^{a}$ & HR $(95 \%$ CI) & $P$ value ${ }^{a}$ & $\mathrm{HR}(95 \% \mathrm{CI})$ & P value ${ }^{a}$ \\
\hline \multicolumn{7}{|l|}{ Before PSM $(N=427)$} \\
\hline Unadjusted model & $2.542(1.532-4.218)$ & $<0.001$ & $1.867(1.276-2.731)$ & 0.001 & $2.704(1.622-4.507)$ & $<0.001$ \\
\hline Adjusted model ${ }^{b}$ & $2.253(1.346-3.773)$ & 0.002 & $1.753(1.190-2.583)$ & 0.005 & 2.483 (1.475-4.179) & 0.001 \\
\hline \multicolumn{7}{|l|}{ After PSM $(N=356)$} \\
\hline Unadjusted model & $2.148(1.256-3.675)$ & 0.005 & $1.684(1.118-2.535)$ & 0.013 & $2.065(1.220-3.495)$ & 0.007 \\
\hline Adjusted model ${ }^{b}$ & $2.058(1.200-3.528)$ & 0.009 & 1.663 (1.103-2.507) & 0.015 & $2.042(1.203-3.464)$ & 0.008 \\
\hline
\end{tabular}

Abbreviations: CI, confidence interval; DMFS, distant metastasis-free survival; HR, hazard ratio; OS, overall survival; PFS, progression-free survival; PSM=propensity score matching; Ref, reference. Bolding shows $P$ value $<0.05$.

aCox proportional hazards model, $\mathrm{P}<0.05$ were in bold.

bAdjusted for sex (male versus female), age (<60 years versus $\geq 60$ years), tumor classification (T1-T2 versus T3-T4), nodal classification (N0-N1 versus N2-N3) and radiotherapy dose (continuous variable).

A number of studies have identified the prognostic value of SLDH level in different types of cancer ${ }^{18-20}$. In several cancers, SLDH level plays even more important role in predicting long-term survival. The International Prognostic Index (IPI) is a common clinical tool used to predict outcomes in patients with non-Hodgkin's lymphoma ${ }^{21}$. It consists of 5 risk factors including elevated SLDH level. Moreover, SLDH level is also included in 2009 AJCC TNM staging system for melanoma ${ }^{22}$. SLDH level is one of secondary determinants of $\mathrm{M}$ staging.

The prognostic value of SLDH on survival in patients with NPC was first studied in 1993 by Chatani et $\mathrm{al}^{23}$. The study showed that SLDH level, together with node involvement of the neck were important prognostic factors for predicting poor outcomes. In China, similar study was first conducted by Liaw et $\mathrm{al}^{24}$. The study reported that SLDH level was higher in metastatic NPC compared to one with local or regional failures. It also found that in comparison to normal pre-treatment SLDH group, high pre-treatment SLDH group had inferior OS (median OS: 10 months versus 53 months, $\mathrm{P}=0.008$ ). In the era of IMRT, several researches were conducted to determine the prognostic value of pre-treatment SLDH level on survival in NPC patients ${ }^{13-15}$. Wei et $\mathrm{al}^{13}$ found that high pre-treatment SLDH $(>171 \mathrm{U} / \mathrm{L}$ in stage I-II and $>225 \mathrm{U} / \mathrm{L}$ in stage III-IV) was an independent prognosticator of inferior overall survival and tumor-free survival. Zhou et $\mathrm{al}^{14}$ 
reported that both pre-treatment and post-treatment SLDH level ( $\leq 245 \mathrm{IU} / \mathrm{L}$ versus $>245 \mathrm{IU} / \mathrm{L})$ are independent prognostic factors of overall survival, disease-free survival and distant metastasis-free survival. Most recently, a study conducted at Fujian Provincial Cancer Hospital ${ }^{15}$ also confirmed the relation of elevated pre-treatment SLDH with poor overall survival, disease-specific survival and distant metastasis-free survival, but no significant correlation found with PFS and locoregional-free survival (LRFS). Although has been studied extensively, most of studies with large patient database came from high endemic areas. This may impend the generalizability of the study due to selection bias and thus, the need for similar studies with large patient samples conducted in areas with significant differences in incidence rates of NPC arises.

The underlying mechanism behind the prognostic value of SLDH has not been clearly understood. However, it has been hypothesized that SLDH level may reflect the extent of hypoxia in tumor cells. In addition, the oxygenation level of a tumor has been reported to be an important determinant of clinical outcomes in patients undergoing radiotherapy and chemotherapy ${ }^{25}$. Due to its rapid proliferation, which leads to low oxygenation, neoplastic cells have its own unique characteristic in term of metabolic pathway. In producing energy, cancer cells mainly use anaerobic glycolysis which enables it to be independent of oxygen supply. This phenomenon is known as Warburg effect ${ }^{26}$. In this situation, lactate dehydrogenase (LDH) is responsible for converting pyruvate to lactate. As the key kinase of this process, the elevated level of LDH ensures the efficiency of this process. Recently, Ding et $\mathrm{al}^{27}$ proposed that the ability of SLDH level in cancer patients was not only because its role in cancer metabolism, but also through altering the tumor microenvironment which led to suppression and evasion of immune system. Therefore, the study suggested elevated SLDH level could also be a new marker of immune suppression in cancer patients.

LDH consists of 2 major subunits: LDH-M and $\mathrm{LDH}-\mathrm{H}$, which can produce 5 different isoenyzmes, including: LDH-1, LDH-2, LDH-3, LDH-4 and LDH-5. Among them, LDH-5 is the most closely related to malignancy $28-30$. With only comprised of LDH-M, LDH-5 plays an important role in anaerobic glycolysis and its level is upregulated in response to low oxygenation microenvironment. Moreover, it has been reported to be associated with the expression of hypoxia-inducible factor $1 a$ (HIF-1a), which activation induced by tumor hypoxia ${ }^{31}$. HIF-1a also alters the expression of tumor suppressor genes expression and regulates tumor angiogenesis ${ }^{31-33}$.
Moreover, previous studies have also confirmed that high expression of HIF-1a contributed to chemoradioresistance in several cancers ${ }^{34,35}$. All of this is beneficial for tumor progression. Taken together, SLDH level may reflect the expression of HIF-1a and subsequent molecular regulation which lead to poor prognosis in malignancy. However, SLDH cannot sufficiently indicate the intratumoral LDH activity. This is likely due to the bulk of the tumor and/or the difference in $\mathrm{LDH}$ clearance between individuals ${ }^{28}$. Koukourakis et al ${ }^{28}$ showed that immunochemistry allowed the assessment of LDH-5 activity in individual cancer cells. It is also reported that both immunochemistry method and SLDH showed positive trend in comparative analysis and the results were not overlapping.

In present study, we provide further confirmation of the prognostic value of pre-treatment SLDH in patients with NPC undergoing IMRT-based therapy. We found that high pre-treatment SLDH was an independent prognostic factor of poor survival in these patients, specifically in terms of OS, PFS and DMFS. In our additional data analysis, however, we did not observed a significant correlation between high pre-treatment SLDH and poor LRFS (5-year LRFS: $84.0 \%$ versus $90.0 \%$, log-rank test: $\mathrm{P}=0.058$, survival curve not shown). Our results are in line with similar studies conducted in Southern China ${ }^{13-15}$. In fact, since our patients had different demographic characteristics compared to previous studies, our present study may improve the generalizability of the role of pre-treatment SLDH in predicting survival of NPC patients.

The strengths of our study included to be the first study conducted in Eastern China with large patient population. Secondly, the cutoff value of pre-treatment SLDH was determined through ROC curve analysis, resulting in appropriate balance between sensitivity and specificity. This approach is different in comparison with previous studies'13-16, which simply used the upper limit of normal SLDH level. We surmise that this approach was not appropriate since the normal range of SDLH level is determined on the basis of healthy individuals and therefore may need to be reevaluated in case of NPC patients. Lastly, we performed propensity score matching analysis, which is an effective approach to adjust bias. Austin ${ }^{36}$ described that propensity score grants one to design and analyze observational study to mimic some important characteristics of a randomized controlled trial. To the best of our knowledge, this approach was never performed in this field before. Thus, we are confident with the results obtained.

However, this study also had several limitations. 
First, since it was a retrospective study and all patients were enrolled from single institution, further large prospective randomized clinical trial in multicenter setting should be conducted to confirm the prognostic impact of pre-treatment SLDH level in NPC patients undergoing IMRT. Moreover, the level LDH-5 was not analyzed specifically in this study, but a previous study conducted by Koukourakis 28 reported that the expression of $\mathrm{LDH}-5$ had positive correlation with the total SLDH level.

\section{Conclusion}

In summary, pre-treatment SLDH level was found to be a useful prognosticator for survival in NPC patients treated with IMRT. As part of routinely performed test and due to its cost-effectiveness, pre-treatment SLDH level can be combined with conventional TNM staging system in determining treatment strategies and predicting prognosis of NPC patients. This way, we can achieve better personalized treatment for NPC patients receiving IMRT. However, our study cannot be considered conclusive and thus, further prospective randomized clinical trial is necessary.

\section{Abbreviations}

AJCC: American Joint Committee on Cancer; CI: confidence interval; CRT: combined chemoradiotherapy; CT: computed tomography; CTV: clinical target volume; DMFS: distant metastasis-free survival; GTVnd: gross tumor volume of metastatic neck lymph nodes; GTVnx: gross tumor volume of nasopharynx; Gy: Gray; HR: hazard ratio; HNC: head and neck cancer; IMRT: intensity-modulated radiotherapy; IPI: international prognostic index; KPS: Karnofsky performance score; L: liter; LDH: lactate dehydrogenase; LRFS: locoregional-free survival; MRI: magnetic resonance imaging; NPC: nasopharyngeal carcinoma; OS: overall survival; PFS: progression-free survival; PSM: propensity score matching; PTV: planning target volume; Ref: reference; ROC: receiver operating characteristics; SD: standard deviation; SLDH: serum lactate dehydrogenase; SPECT: single-photon emission computed tomography; TNM: tumor-node-metastasis; U: unit.

\section{Competing Interests}

The authors have declared that no competing interest exists.

\section{References}

1. Torre LA, Bray F, Siegel RL, Ferlay J, Lortet-Tieulent J, Jemal A. Global cancer statistics, 2012. CA Cancer J Clin. 2015; 65(2): 87-108.

2. Cao SM, Simons MJ, Qian CN. The prevalence and prevention of nasopharyngeal carcinoma in China. Chin J Cancer. 2011; 30(2): 114-9.
3. Huang PY, Zeng Q, Cao KJ, Guo X, Guo L, Mo HY, Wu PH, Qian CN, Mai HQ, Hong $\mathrm{MH}$. Ten-year outcomes of a randomised trial for locoregionally advanced nasopharyngeal carcinoma: A single-institution experience from an endemic area. Eur J Cancer. 2015; 51(13) :1760-70.

4. Yi JL, Gao L, Huang XD, Li SY, Luo JW, Cai WM, Xiao JP, Xu GZ. Nasopharyngeal carcinoma treated by radical radiotherapy alone: Ten-year experience of a single institution. Int J Radiat Oncol Biol Phys. 2006; 65(1): 161-8.

5. Kong F, Ying H, Huang S, Du C, Zhou J, Hu C. Preliminary results of nasopharyngeal carcinoma treated with intensity-modulated radiotherapy: a retrospective study of 364 patients. Eur Arch Otorhinolaryngol. 2014; 271(12): 3283-90.

6. Zhang MX, Li J, Shen GP, Zou X, Xu JJ, Jiang R, You R, Hua YJ, Sun Y, Ma J, Hong MH, Chen MY. Intensity-modulated radiotherapy prolongs the survival of patients with nasopharyngeal carcinoma compared with conventional two-dimensional radiotherapy: A 10-year experience with a large cohort and long follow-up. Eur J Cancer. 2015; 51(17): 2587-95.

7. Sun X, Su S, Chen C, Han F, Zhao C, Xiao W, Deng X, Huang S, Lin C, Lu T. Long-term outcomes of intensity-modulated radiotherapy for 868 patients with nasopharyngeal carcinoma: an analysis of survival and treatment toxicities. Radiother Oncol. 2014; 110(3): 398-403.

8. Li AC, Xiao WW, Shen GZ, Wang L, Xu AA, Cao YQ, Huang SM, Lin CG, Han F, Deng XW, Zhao C. Distant metastasis risk and patterns of nasopharyngeal carcinoma in the era of IMRT: long-term results and benefits of chemotherapy. Oncotarget. 2015; 6(27): 24511-21.

9. Lee AW, Ng WT, Chan LK, Chan OS, Hung WM, Chan CC, Cheng PT, Sze H, Lam TS, Yau TK. The strength/weakness of the AJCC/UICC staging system (7th edition) for nasopharyngeal cancer and suggestions for future improvement. Oral Oncol. 2012; 48(10): 1007-13.

10. Tang LQ, Chen QY, Guo SS, Chen WH, Li CF, Zhang L, Lai XP, He Y, Xu YX, Hu DP, Wen SH, Peng YT, Liu H, Liu LT, Yan SM, Guo L, Zhao C, Cao KJ, Liu Q, Qian CN, Ma J, Guo X, Zeng MS, Mai HQ. The impact of plasma Epstein-Barr virus DNA and fibrinogen on nasopharyngeal carcinoma prognosis: an observational study. Br J Cancer. 2014; 111(6): 1102-11.

11. Zhang W, Chen Y, Chen L, Guo R, Zhou G, Tang L, Mao Y, Li W, Liu X, Du X, Sun Y, Ma J. The clinical utility of plasma Epstein-Barr virus DNA assays in nasopharyngeal carcinoma: the dawn of a new era?: a systematic review and meta-analysis of 7836 cases. Medicine (Baltimore). 2015; 94(20): e845.

12. Wan XB, Fan XJ, Chen MY, Xiang J, Huang PY, Guo L, Wu XY, Xu J, Long ZJ, Zhao Y, Zhou WH, Mai HQ, Liu Q, Hong MH. Elevated Beclin 1 expression is correlated with HIF-1alpha in predicting poor prognosis of nasopharyngeal carcinoma. Autophagy. 2010; 6(3): 395-404.

13. Wei Z, Zeng X, Xu J, Duan X, Xie Y. Prognostic value of pretreatment serum levels of lactate dehydrogenase in nonmetastatic nasopharyngeal carcinoma: single-site analysis of 601 patients in a highly endemic area. Onco Targets Ther. 2014; 7: 739-49.

14. Zhou GQ, Ren XY, Mao YP, Chen L, Sun Y, Liu LZ, Li L, Lin AH, Mai HQ, Ma J. Prognostic implications of dynamic serum lactate dehydrogenase assessments in nasopharyngeal carcinoma patients treated with intensity-modulated radiotherapy. Sci Rep. 2016; 6: 22326.

15. Chen Z, Guo Q, Lu T, Lin S, Zong J, Zhan S, Xu L, Pan J. Pretreatment Serum Lactate Dehydrogenase Level as an Independent Prognostic Factor of Nasopharyngeal Carcinoma in the Intensity-Modulated Radiation Therapy Era. Med Sci Monit. 2017; 23: 437-445.

16. Zhou GQ, Tang LL, Mao YP, Chen L, Li WF, Sun Y, Liu LZ, Li L, Lin AH, Ma J. Baseline serum lactate dehydrogenase levels for patients treated with intensity-modulated radiotherapy for nasopharyngeal carcinoma: a predictor of poor prognosis and subsequent liver metastasis. Int J Radiat Oncol Biol Phys. 2012; 82(3): e359-65.

17. Moretto F, Rampino M, Munoz F, Ruo Redda MG, Reali A, Balcet V, Badellino S, Piva C, Schena M, Airoldi M, Ostellino O, Pecorari G, Ragona R, Ricardi U. Conventional 2D (2DRT) and 3D conformal radiotherapy (3DCRT) versus intensity-modulated radiotherapy (IMRT) for nasopharyngeal cancer treatment. Radiol Med. 2014; 119(8): 634-41.

18. Agrawal A, Gandhe MB, Gupta D, Reddy MV. Preliminary Study on Serum Lactate Dehydrogenase (LDH)-Prognostic Biomarker in Carcinoma Breast. J Clin Diagn Res. 2016; 10(3): BC06-8.

19. Zhu L, Li X, Shen Y, Cao Y, Fang X, Chen J, Yuan Y. A new prognostic score based on the systemic inflammatory response in patients with inoperable non-small-cell lung cancer. Onco Targets Ther. 2016; 9: 4879-86.

20. Yu SL, Xu LT, Qi Q, Geng YW, Chen H, Meng ZQ, Wang P, Chen Z. Serum lactate dehydrogenase predicts prognosis and correlates with systemic inflammatory response in patients with advanced pancreatic cancer after gemcitabine-based chemotherapy. Sci Rep. 2017; 7: 45194.

21. International Non-Hodgkin's Lymphoma Prognostic Factors Project. A predictive model for aggressive non-Hodgkin's lymphoma. N Engl J Med. 1993; 329(14): 987-94

22. Balch CM, Gershenwald JE, Soong SJ, Thompson JF, Atkins MB, Byrd DR, Buzaid AC, Cochran AJ, Coit DG, Ding S, Eggermont AM, Flaherty KT, Gimotty PA, Kirkwood JM, McMasters KM, Mihm MC Jr, Morton DL, Ross MI, Sober AJ, Sondak VK. Final version of 2009 AJCC melanoma staging and classification. J Clin Oncol. 2009; 27(36): 6199-206.

23. Chatani M, Matayoshi Y, Masaki N, Fujii T, Umatani K, Yoshino K, Sato T. Radiation therapy of the nasopharyngeal carcinoma. Treatment results and prognostic factors. Strahlenther Onkol. 1993; 169(9): 527-33. 
24. Liaw CC, Wang CH, Huang JS, Kiu MC, Chen JS, Chang HK. Serum lactate dehydrogenase level in patients with nasopharyngeal carcinoma. Acta Oncol. 1997; 36(2): 159-64

25. Durand RE. Keynote address: the influence of microenvironmental factors on the activity of radiation and drugs. Int J Radiat Oncol Biol Phys. 1991; 20(2): 253-8.

26. Warburg, O. On the Origin of Cancer Cells. Science. 1956; 123(3191): 309-314.

27. Ding J, Karp JE, Emadi A. Elevated lactate dehydrogenase (LDH) can be a marker of immune suppression in cancer: Interplay between hematologic and solid neoplastic clones and their microenvironments. Cancer Biomark. 2017; 19(4): 353-363.

28. Koukourakis MI, Giatromanolaki A, Sivridis E, Bougioukas G, Didilis V, Gatter KC, Harris AL; Tumour and Angiogenesis Research Group. Lactate dehydrogenase-5 (LDH-5) overexpression in non-small-cell lung cancer tissues is linked to tumour hypoxia, angiogenic factor production and poor prognosis. Br J Cancer. 2003; 89(5): 877-85.

29. Zhao $\mathrm{YH}$, Zhou M, Liu $\mathrm{H}$, Ding $\mathrm{Y}$, Khong HT, Yu D, Fodstad O, Tan $\mathrm{M}$. Upregulation of lactate dehydrogenase A by ErbB2 through heat shock factor 1 promotes breast cancer cell glycolysis and growth. Oncogene. 2009; 28(42): 3689-701.

30. Giatromanolaki A, Sivridis E, Gatter KC, Turley H, Harris AL, Koukourakis MI; Tumour and Angiogenesis Research Group. Lactate dehydrogenase 5 (LDH-5) expression in endometrial cancer relates to the activated VEGF/VEGFR2(KDR) pathway and prognosis. Gynecol Oncol. 2006; 103(3): 912-8.

31. Harris AL. Hypoxia--a key regulatory factor in tumour growth. Nat Rev Cancer. 2002 Jan;2(1):38-47. Review. PubMed PMID: 11902584.

32. Colgan SM, Mukherjee S, Major P. Hypoxia-induced lactate dehydrogenase expression and tumor angiogenesis. Clin Colorectal Cancer. 2007; 6(6): 442-6.

33. Ajdukovic J. HIF-1--a big chapter in the cancer tale. Exp Oncol. 2016; 38(1): 9-12.

34. Wan XB, Fan XJ, Chen MY, Xiang J, Huang PY, Guo L, Wu XY, Xu J, Long ZJ, Zhao Y, Zhou WH, Mai HQ, Liu Q, Hong MH. Elevated Beclin 1 expression is correlated with HIF-1alpha in predicting poor prognosis of nasopharyngeal carcinoma. Autophagy. 2010; 6(3): 395-404.

35. Zhou M, Zhao Y, Ding Y, Liu H, Liu Z, Fodstad O, Riker AI, Kamarajugadda S, Lu J, Owen LB, Ledoux SP, Tan M. Warburg effect in chemosensitivity: targeting lactate dehydrogenase-A re-sensitizes taxol-resistant cancer cells to taxol. Mol Cancer. 2010; 9: 33

36. Austin PC. An Introduction to Propensity Score Methods for Reducing the Effects of Confounding in Observational Studies. Multivariate Behav Res. 2011; 46(3): 399-424. 